\title{
Complex Skull Base Reconstructions in Kadish D Esthesioneuroblastoma: Case Report
}

\author{
Sheri K. Palejwala ${ }^{1}$ Saurabh Sharma ${ }^{2}$ Christopher H. Le ${ }^{2} \quad$ Eugene Chang ${ }^{2}$ Audrey B. Erman ${ }^{2}$ \\ G. Michael Lemole, J ${ }^{1}$
}

${ }^{1}$ Division of Neurosurgery, Department of Surgery, University of Arizona, Tucson, Arizona, United States

${ }^{2}$ Department of Otolaryngology, University of Arizona, Tucson,

Arizona, United States

Address for correspondence Sheri K. Palejwala, MD, Division of Neurosurgery, Department of Surgery, University of Arizona, $1501 \mathrm{~N}$. Campbell Ave., Tucson, AZ 85724-5070, United States (e-mail: Spalejwala@email.arizona.edu).

J Neurol Surg Rep 2017;78:e86-e92.

\begin{abstract}
Introduction Advanced Kadish stage esthesioneuroblastoma requires more extensive resections and aggressive adjuvant therapy to obtain adequate disease-free control, which can lead to higher complication rates. We describe the case of a patient with Kadish D esthesioneuroblastoma who underwent multiple surgeries for infectious, neurologic, and wound complications, highlighting potential preventative and salvage techniques.

Case Presentation A 61-year-old man who presented with a large left-sided esthesioneuroblastoma, extending into the orbit, frontal lobe, and parapharyngeal nodes. He underwent margin-free endoscopic-assisted craniofacial resection with adjuvant

\section{Keywords}

- esthesioneuroblastoma

- olfactory neuroblastoma

- skull base reconstruction

- cerebrospinal fluid leak

- pneumocephalus

- sinonasal malignancy

- complications craniofacial and cervical radiotherapy and concomitant chemotherapy. He then returned with breakdown of his skull base reconstruction and subsequent frontal infections and ultimately received 10 surgical procedures with surgeries for infectionrelated issues including craniectomy and abscess evacuation. He also had surgeries for skull base reconstruction and CSF leak, repaired with vascularized and free autologous grafts and flaps, synthetic tissues, and CSF diversion.

Discussion Extensive, high Kadish stage tumors necessitate radical surgical resection, radiation, and chemotherapy, which can lead to complications. Ultimately, there are several options available to surgeons, and although precautions should be taken whenever possible, risk of wound breakdown, leak, or infection should not preclude radical surgical resection and aggressive adjuvant therapies in the treatment of esthesioneuroblastoma.
\end{abstract}

\section{Introduction}

Esthesioneuroblastoma, or olfactory neuroblastoma, was first described by Berger et al, in 1924 as L'esthésioneuroépithéliome olfactif. $^{1}$ Since then several hundred patients have been described in case series, single-institution reviews, and meta-analyses. ${ }^{2-26}$ Patient presentation is often nonspecific, including congestion and sinusitis-like symptoms, which makes diagnosis challenging, such that most patients have advanced disease at the time of diagnosis. ${ }^{15,18,27}$ The gold standard for the treatment of esthesioneuroblastomas is craniofacial resection with histologically proven disease-free margins, with the use of radiotherapy. $2,7,8,10,12,20,25$ The use of chemotherapy, neoadjuvant therapy, neck dissection, and irradiation, however, remains controversial. ${ }^{5-7,11,14,22}$

Esthesioneuroblastoma has an established propensity for being locally aggressive with the possibility of distal metastases and leading to decades-delayed recurrences., ${ }^{5,11,12,20}$ In received

November 11, 2016 accepted after revision March 4, 2017
DOI https://doi.org/

10.1055/s-0037-1601877. ISSN 2193-6366. (c) 2017 Georg Thieme Verlag KG
Stuttgart • New York

License terms

$\Theta(1) \Theta \Theta$ 
an effort to prolong disease-free survival, most advocate for radical resection with clear margins, which has been shown to double disease-free survival, especially in the setting of advanced Kadish tumors. ${ }^{2,5,7,28}$ Additionally, greater tumor extent often necessitates substantial resections, which have both been associated with complication rates as high as $33 \%{ }^{28-30}$ More extensive disease, meticulous surgical resection, and aggressive adjuvant therapies together increase the likelihood of adverse events, including cerebrospinal fluid (CSF) leak, neurologic deficits, and infectious complications.

We performed a retrospective chart review to describe the history of a single patient with Kadish D esthesioneuroblastoma, who underwent endoscopic-assisted craniofacial resection followed by adjuvant radiation and chemotherapy. His course was complicated by multiple infections and CSF leaks necessitating several skull base reconstructions. We describe his case to elucidate the multiple and successive escalations for reconstruction available to skull base surgeons, even in the face of malignancy, radiation, chemotherapy, and infection.

\section{Case Report}

A 61-year-old man, with a several decade history of chronic sinusitis, presented with a 5-month history of nasal congestion, decreased sense of taste and smell, and intermittent yellow nasal drainage, which failed to resolve with antibiotics, scant epistaxis, left ptosis, and medial periorbital edema. Nasal endoscopy revealed a 7-cm nasal mass, and biopsy demonstrated esthesioneuroblastoma. On imaging there was a homogenously enhancing, erosive left skull base lesion with extension into the right nasal cavity, bilateral ethmoid, left maxillary, frontal, and sphenoid sinuses with anterior cranial fossa extension and leptomeningeal enhancement (- Fig. 1). Two mildly enlarged fludeoxyglucose (FDG)-avid left parapharyngeal lymph nodes were seen on positron emission tomography-computed tomography (PET-CT).

He subsequently underwent a combined level 1 transbasal and endoscopic endonasal approach for complete, margin-free resection. At the start of the procedure, the bilateral middle and superior turbinates were removed, and tumor was resected from the nasal cavity, ethmoid and sphenoid sinuses, and frontal recess. This was followed by a transbasal level 1 craniotomy with removal of the medial orbital bar and both intra- and extradural tumor resection and resection of the skull base dura. All margins were negative and reconstruction was performed with a large vascularized pericranial flap onlay, four standard and two firm Nasopores (Polyganics, the Netherlands), and nasal trumpets. The patient was discharged home on postoperative day (POD) 5 without any perioperative complications.

The patient then received intensity-modulated radiation therapy (IMRT) with $54 \mathrm{~Gy}$ to the tumor bed and $70 \mathrm{~Gy}$ for his cervical lymph node disease, with two cycles of cisplatin and etoposide concurrently. After completing his therapy, 3.5 months after surgery, he had no radiographic evidence of residual or recurrent disease (-Fig. 2). PET-CT 6 months after surgery revealed decreased size and activity of the parapharyngeal nodes as well. His postoperative course was

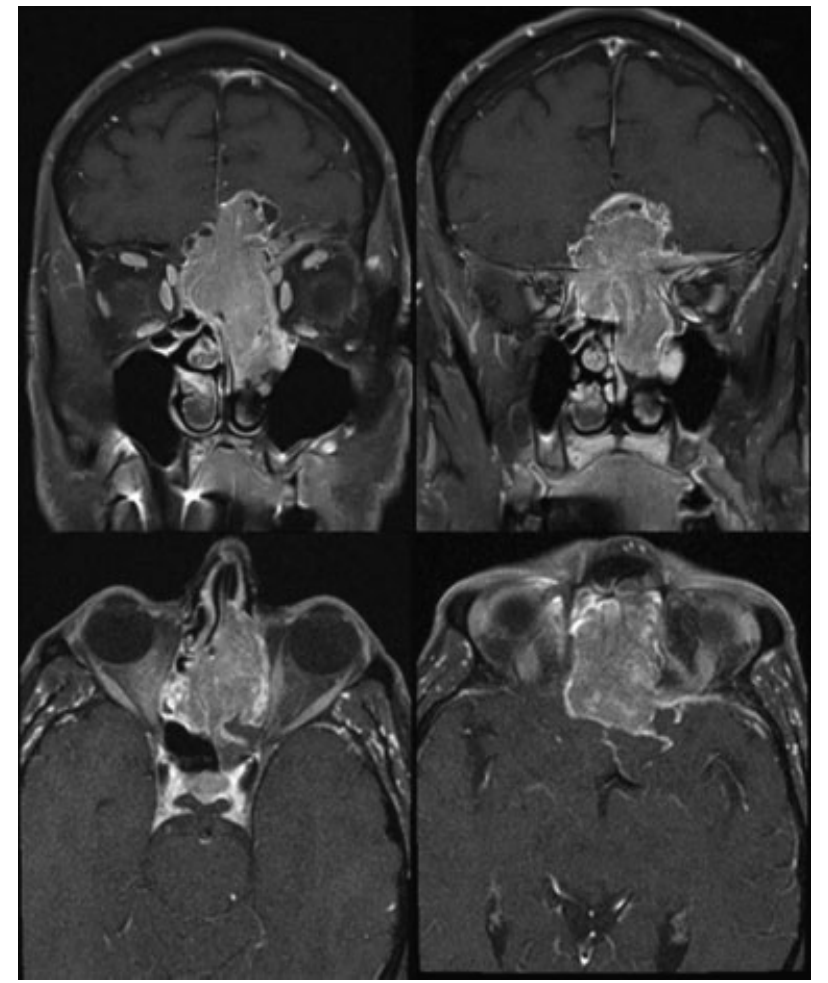

Fig. 1 Coronal and axial T1-weighted contrasted MRI showing large, avidly enhancing skull base mass centered in the left nasal cavity. The lesion abuts and laterally displaces the left orbit and has extensive intracranial invasion with leptomeningeal enhancement but without evidence of cavernous sinus invasion.

complicated by radiation-induced dysphagia, treated with temporary percutaneous endoscopic gastrostomy (PEG) tube placement, and nasal crusting, managed with periodic debridements and antibiotics when indicated.

He was regularly followed by the neurosurgical, otolaryngology, radiation, and medical oncology teams. Nearly 1 year after surgery, he presented with progressive left eye ptosis and painful forehead and left canthal lesions. Magnetic resonance imaging (MRI) showed avid enhancement and a neofrontal sinus in the epidural space (-Fig. 3). He was taken to the operating room 15 months after his initial resection for endoscopic debridement, biopsies to rule out intranasal recurrence, and fine-needle aspiration (FNA) of the forehead masses. He was found to have significant mucopurulence, osteoradionecrosis of portions of his frontal bone, which were then removed, and shrinking of his pericranial flap with an airspace between the pericranium and frontal bone ( - Fig. 4). He then underwent FNA of the forehead mass and left endoscopic dacryocystorhinostomy (DCR). No malignancy was found, but forehead cultures grew Proteus mirabilis and Klebsiella pneumoniae for which he was treated with intravenous (IV) metronidazole and ceftriaxone for 8 weeks. After extensive multidisciplinary discussions, the decision was made to proceed with removal of the necrotic bone flap.

Seventeen months after initial resection, the patient underwent craniectomy, irrigation and debridement, titanium mesh cranioplasty, and skull base reconstruction with an autologous fascia lata graft. There was no gross 


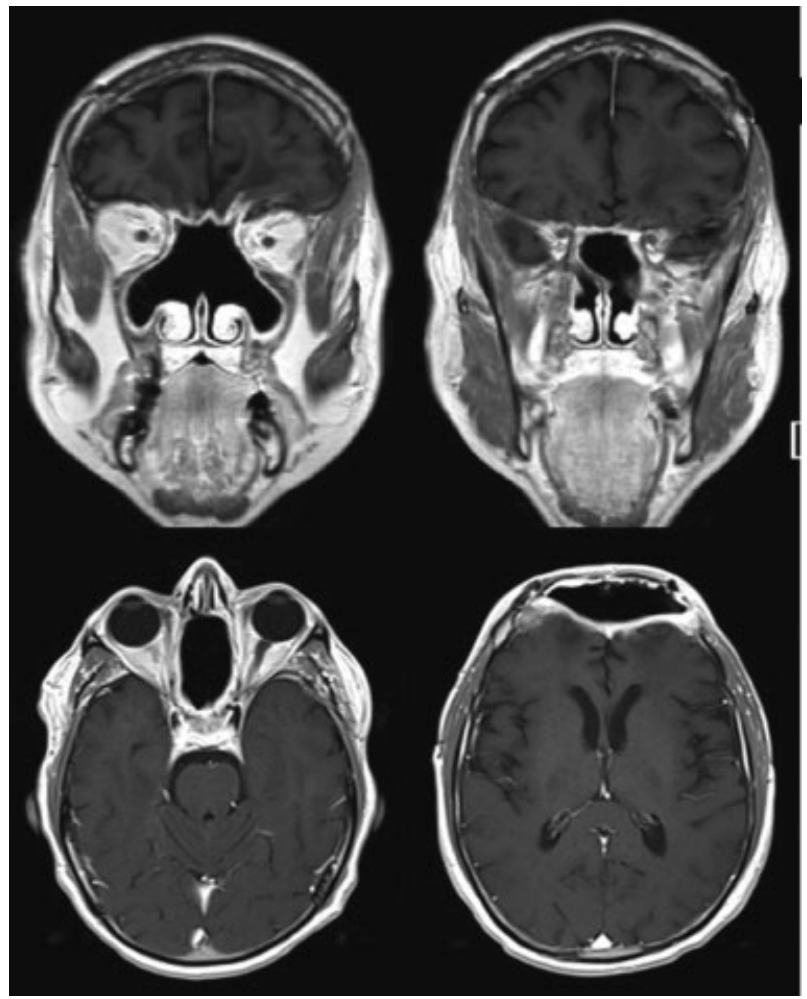

Fig. 2 Coronal and axial T1-weighted contrasted MRI 3.5 months after surgery and concurrent radiation and chemotherapy showing extensive resection without evidence of residual or recurrent disease.

extracranial infection, but an obvious epidural collection with fibrinous exudate, and osteomyelitis and necrosis of the orbital bar. Both were removed and discarded. We also encountered a focal dehiscence in the pericranium, allowing communication between the endonasal and intracranial spaces. This was repaired with a water-tight closure using a fascia lata autograft. After extensive irrigation and debridement, cranial reconstruction was performed with titanium mesh, which was contoured to the nasal bridge and provided medial orbital bar reconstruction. No evidence of malignancy was noted either grossly or on histopathology.
He was discharged home on POD 5 with 4 weeks of IV metronidazole and ceftriaxone. Cultures revealed Mycobacterium chelonae for which he was treated with additional IV amikacin, IV tigecycline, and PO clarithromycin for an additional 2 weeks, and then continued on PO doxycycline and clarithromycin for 12 weeks.

Four months later, CT revealed a persistent intracranial air pocket with an area of presumed continued nasal-intracranial communication. After completing his antibiotic regimen, the patient underwent cranial reconstruction with a custom polyetheretherketone (PEEK) bone flap with an orbital bar extension and vascularized left radial forearm free flap, to cover the anterior skull base defect, 5 months after the craniectomy (22 months after initial surgery). Reconstruction required partial takedown of the pericranial flap laterally to access the skull base posteriorly to the level of the planum sphenoidale. We encountered a small area of CSF leak around the left orbit that was reconstructed using primary closure and Tisseel fibrin sealant (Baxter International Inc., Deerfield, Illinois, United States). The vascularized radial forearm myofascial flap was anastomosed to the superficial temporal artery and vein and was found to be well perfused and laying freely. It was secured to the dura and skull base, and nasal endoscopy confirmed complete skull base closure. Postoperative imaging is seen in - Fig. $\mathbf{5}$. On POD 2 the patient experienced acute altered mental status and disinhibition, CT revealed a large bifrontal epidural hematoma, and the patient was taken emergently to the operating room for evacuation (-Fig. 6). Another small CSF leak was noted and also repaired with primary closure, Tisseel fibrin sealant, and placement of a lumbar drain. His mental status gradually returned to baseline and he was discharged home on POD 7.

He returned to the emergency department 2 days after discharge after reports of strange disinhibited behavior. CT revealed intracranial air with a new endonasal-intracranial focus of communication, suspicious for tension pneumocephalus (-Fig. 7). He was taken to the operating room for yet another skull base reconstruction where the new defect was clearly noted. An AlloDerm (LifeCell Corp., Woodlands, Texas,
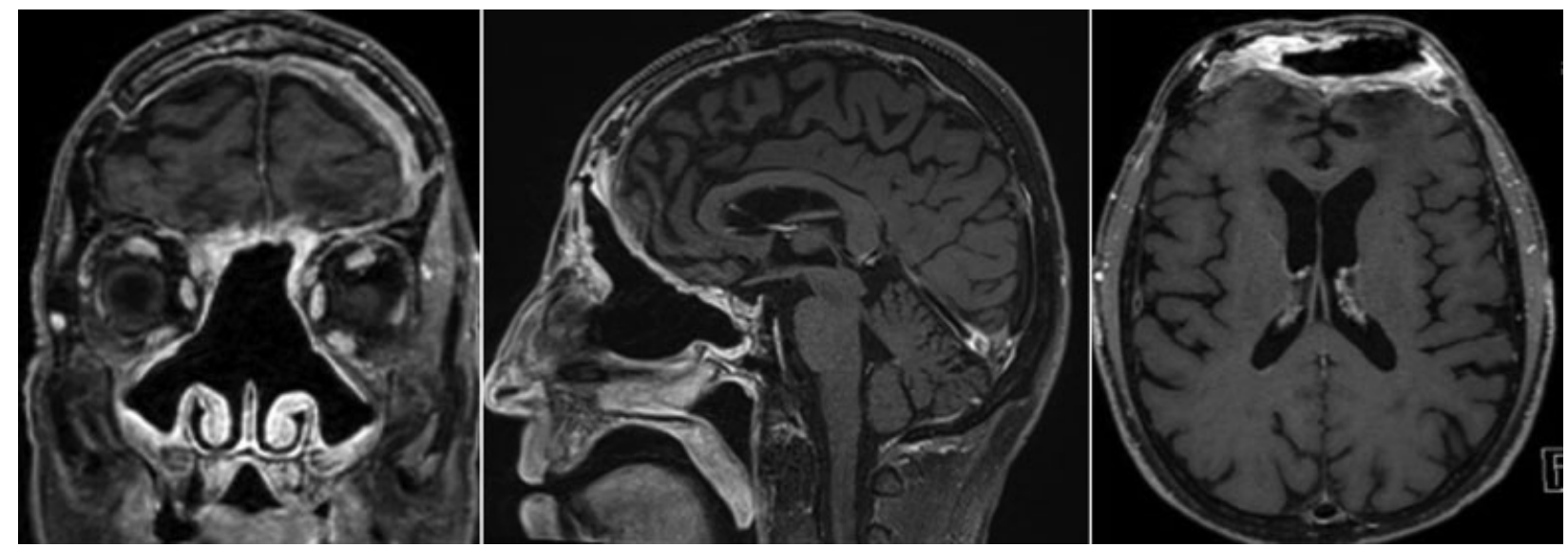

Fig. 3 Coronal, sagittal, and axial, respectively, T1-weighted contrasted MRI demonstrating frontal bone osteoradionecrosis with creation of a neofrontal sinus within the epidural space and enhancement concerning for infection. 


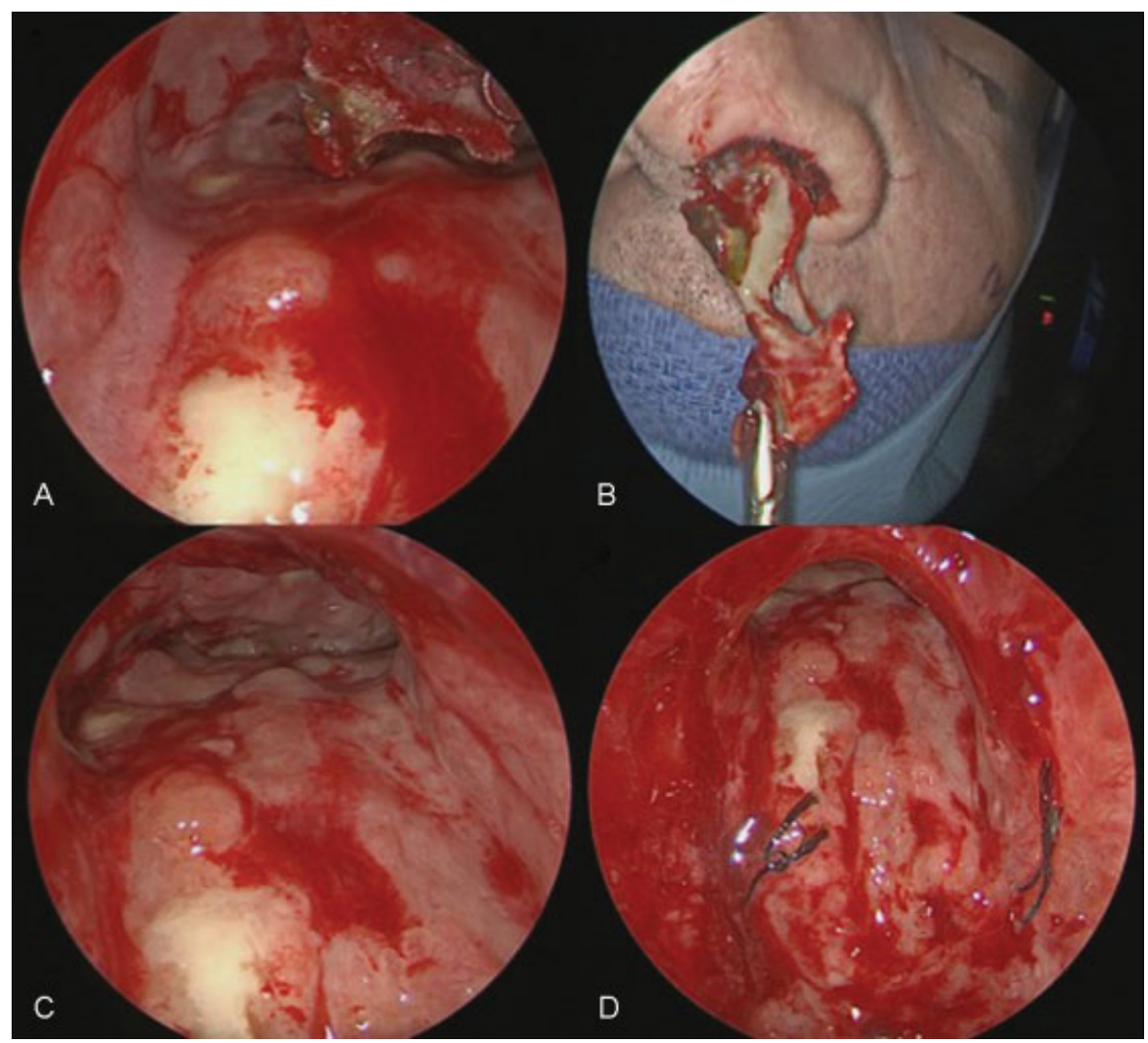

Fig. 4 Intraoperative endoscopic images showing frontal sinus mucopurulence and osteonecrotic bone, as well as defect in pericranial flap coverage over the right-sided anterior skull base.

United States) graft was cut to size and sutured both to the planum sphenoidale and the periorbita bilaterally. Additional AlloDerm was used as an on-lay over the friable frontal lobe dura and secured with Evicel fibrin sealant (Johnson \& Johnson Wound Management, Somerville, New Jersey, United States). The myofascial flap was reapproximated and reconstruction was again confirmed with nasal endoscopy (-Fig. 8). He had a gradual return to his intact neurologic baseline and was discharged home on POD 9.
The patient returned 1 month later to the emergency department (ED) with complaints of 2 days of severe progressive headache and subgaleal fluid collection. He was treated with 5 days of CSF drainage via a lumbar drain and discharged on hospital day 8 , after observation for two days after drain removal. Unfortunately, he returned with recurrence of the subgaleal fluid collection, and moderate amount of fluid expressed from a forehead pustule. Imaging revealed increase in the subgaleal and epidural fluid, and he was once

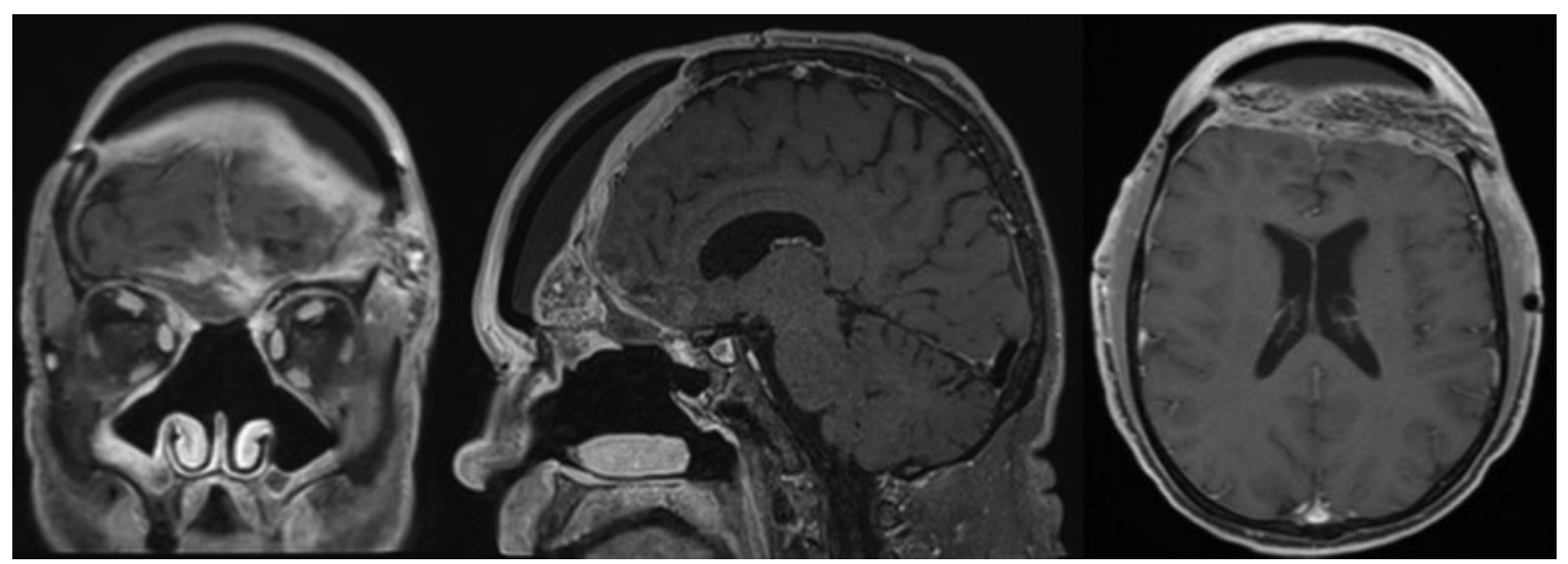

Fig. 5 Coronal, sagittal, and axial, respectively, T1-weighted contrasted MRI performed immediately after skull base reconstruction, vascularized myofascial flap, and PEEK cranioplasty. 


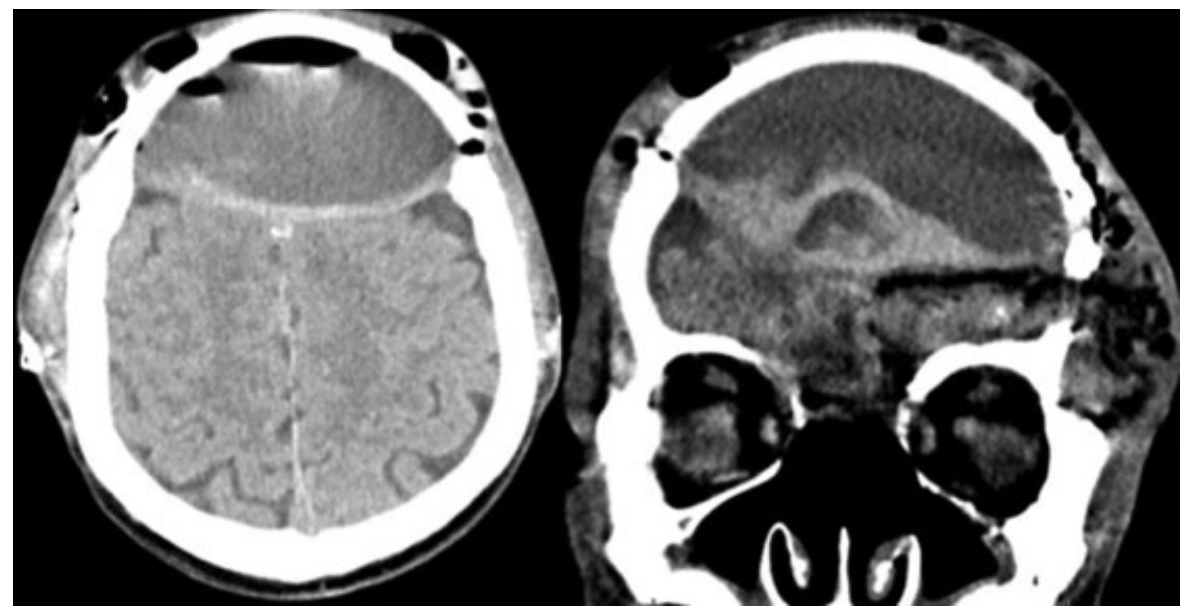

Fig. 6 Axial CT showing a postoperative $5 \mathrm{~cm}$ epidural hematoma with frontal lobe effacement.

again admitted for an epidural-peritoneal shunt, without immediate perioperative complications. However, the epidural fluid culture later returned as Enterobacter cloacae and was treated with 8 total weeks of IV meropenem. He also complained of abdominal pain concerning for infectious or chemical peritonitis, while abdominal CT revealed fat infiltration and a right lower quadrant fluid loculation. He was reluctant to have his shunt removed due to concern for fluid reaccumulation and possible subsequent infection.

Seven weeks after shunt placement, the patient presented to the ED with rhinorrhea and pneumocephalus and was taken for shunt removal and epidural drain placement. Four days later, he underwent yet another skull base reconstruction with repositioning of the still viable myofascial flap, additional buttressing with an abdominal fat graft, and insertion of lumbar drain. There was no evidence of infection or tumor recurrence. His postoperative course was complicated with abdominal hematoma that was evacuated at bedside, and he was discharged home on POD 8.

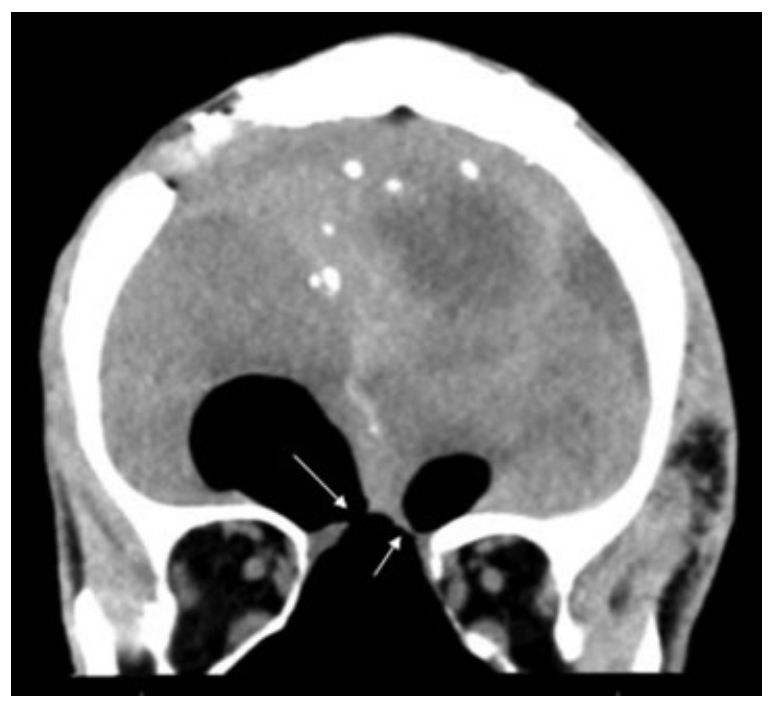

Fig. 7 Coronal CT scan showing two foci (arrows) of breakdown in the skull base reconstruction with extensive bifrontal pneumocephalus.
After the aforementioned 8 weeks of IV meropenem, the patient was transitioned to 4 weeks of PO levofloxacin and continued to be monitored with imaging and clinical nasal endoscopy. MRI performed 26 months after initial resection then revealed right temporoparietal and falcine nodular dural enhancement concerning for metastatic disease (-Fig.9). PETCT revealed progression of his intracranial disease and pulmonary nodules. He was referred to both medical and radiation oncology for salvage adjuvant treatment.

\section{Discussion}

The first, and most commonly used, staging system for esthesioneuroblastoma was described in 1976 by Kadish et al and was later modified by Foote et al to include involvement of cervical lymph node and distant metastases. ${ }^{19}$ High Kadish classification, especially with cervical lymph node involvement, is known to be a profound negative prognosticator, cutting survival in half and increasing the rate of distal

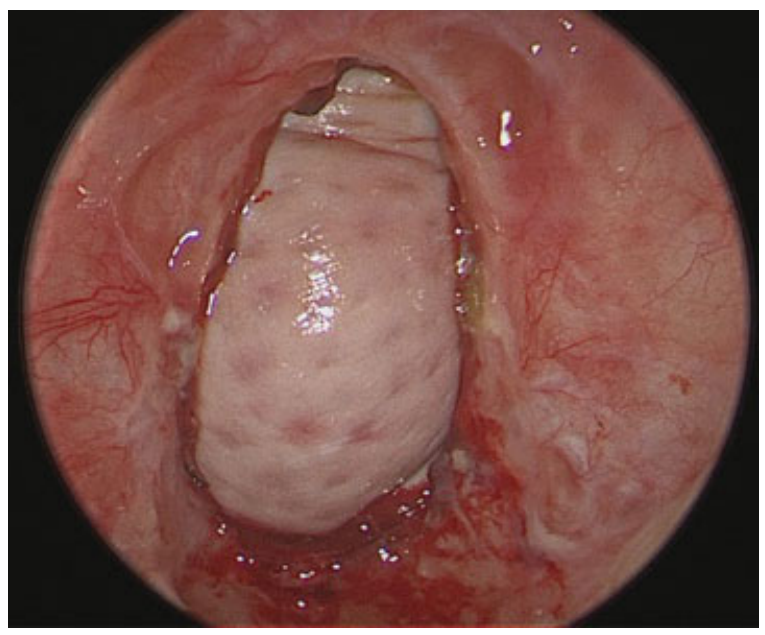

Fig. 8 Intraoperative neuroendoscopic image showing AlloDerm from the skull base reconstruction. 


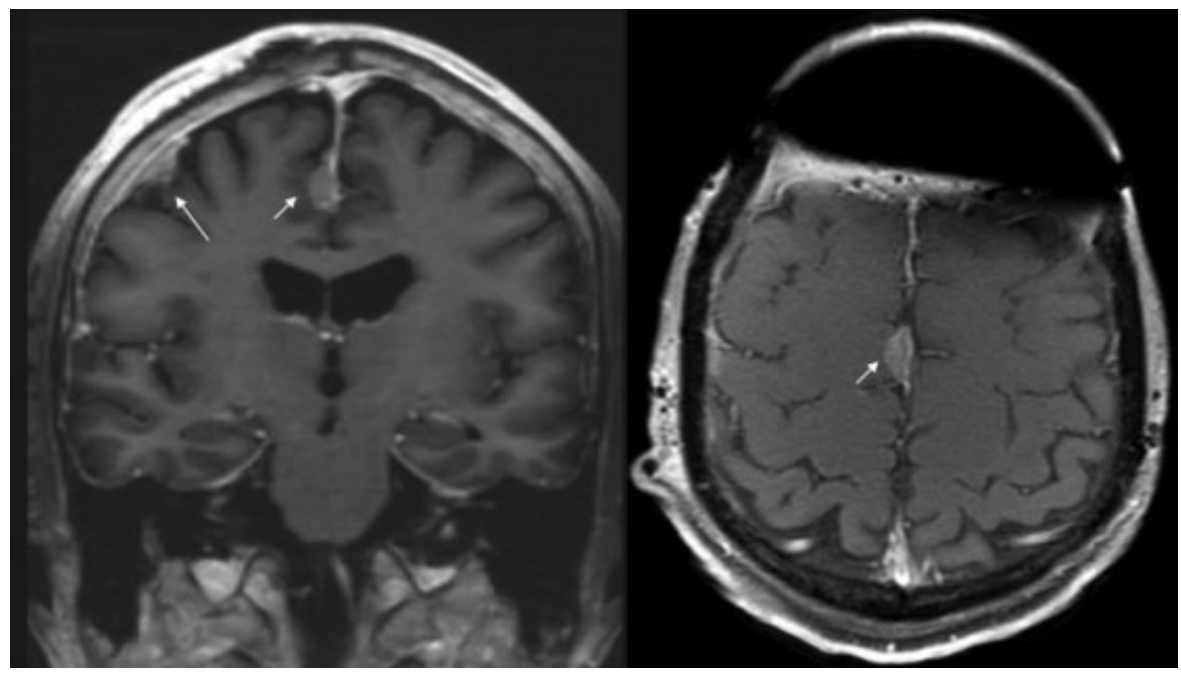

Fig. 9 Coronal and axial T1-weighted contrasted MRI showing the right-sided temporoparietal convexity and parfalcine nodular enhancing lesions (arrows) suspicious for metastatic disease.

metastasis by $35 \%$. ${ }^{4,17,19,23}$ On the other hand, aggressive surgical resection, with clear margins, is a strong predictor of disease freedom. ${ }^{2,7,18,28}$ Anterior skull base tumors with positive margins were found to have double the incidence of local recurrence and half the survival of complete resections. $^{7,28}$ The widespread use of craniofacial approaches has improved our ability to obtain complete resections with histologically disease-free margins, even in the setting of extensive, high Kadish stage tumors. 2,7,10,12,23,25 Craniofacial resection has been shown to increase progression-free survival from 37.5 to $82 \%$. $71,14,23,28$

Despite the favorable outcomes with extensive resection, single-modality treatment, including surgery in isolation, led to poor results and higher rates of both local recurrence and distal metastatic disease. ${ }^{15,18}$ Besides radical resection, the highest control rates were found with the addition of radiation therapy. Dulguerov et al performed a meta-analysis of 390 patients which ultimately showed the best outcomes in esthesioneuroblastoma occurred when margin-free resection was followed by radiotherapy; this has also been corroborated by several large studies. ${ }^{7,12,20}$ Although controversial, many groups argue for the addition of chemotherapy as well, to achieve the longest duration of tumor freedom. ${ }^{18,24}$ Multimodality treatment is especially recommended in those with high Kadish stage esthesioneuroblastoma. ${ }^{15}$

Although aggressive management of these persistent malignant tumors is advocated, there are treatment effects and complications that should be considered. Postoperative complication rates for craniofacial approaches can affect one in three patients undergoing anterior craniofacial resection based on an International Collaborative Study, with wound complications in $18 \%$ of patients. ${ }^{28}$ Although there have been significant advances in skull base reconstruction, especially from endoscopic approaches, CSF leak still remains a significant concern. ${ }^{12,31}$ Some studies advocate the use of synthetics, whereas others use vascularized nasoseptal flaps, and still other groups encourage the use of gasket seals. ${ }^{5,12,31}$ Ultimately there are numerous options available to the surgeon, which can be used simultaneously or even sequentially, should the need arise. Unfortunately, prior radiation treatment and wide intracranial tumor extension increase the risks of complications, including infection and leak. ${ }^{28-30}$

Patients requiring postoperative radiation therapy will likely also incur decreased vascularization and increased fibrosis. ${ }^{32,33}$ Over time, most free flaps, without vascularization, will reabsorb, and these flaps can be salvaged by buttressing them with vascularized flaps from the outset. ${ }^{32}$ Additionally, in the setting of radiation, allografts have been shown to have a high rate of extrusion. ${ }^{32,34}$ Pedicled pericranial flaps are the most frequently used and easily accessible flaps and have been proven successful. ${ }^{34}$ Nasoseptal flaps have also been shown to have tremendous success, even in the setting of wide extensive tumors. ${ }^{31,32}$ However, in the setting of malignancy, they can only be safely used if histologically confirmed to be tumor-free. Unfortunately, in our case, this was not enough to prevent breakdown, with subsequent nasalintracranial communication and an epidural, neofrontal sinus. Pedicled myocutaneous and myofascial flaps have also been used with success, including the pectoralis major, latissimus dorsi, and trapezius muscles. They have both the ability to fill dead space, dampen the effect of CSF and brain pulsations, and have a rich vascular supply. ${ }^{32}$ Although this provided a good salvage reconstruction for our patient, it was not without its own complication, a symptomatic epidural hematoma requiring emergent evacuation. Free tissue transfer and synthetic allografts are also available options, particularly when supplementing more robust vascularized flaps, in the setting of chemotherapy and radiation. This was also corroborated with our own series and experience with this patient.

\section{Conclusion}

Studies have shown that greater tumor extent, especially in the setting of intracranial extension, cervical lymph node involvement, and distal metastases are associated with greater rates of recurrence and poorer prognoses., ${ }^{47,19,23}$ 
In light of this, initial treatment, both medical and surgical, should be aimed at curing the primary disease. Measures should be taken to ensure adequate skull base reconstruction from the outset, as well as any risk mitigating actions that can be performed. Nevertheless, sequential escalation of varied repair techniques is possible and often necessary in these challenging cases. Early use of vascularized flaps should be considered after aggressive skull base resections, radiation, and chemotherapy. Ultimately, there are several options available to surgeons, and although precautions should be taken whenever possible, risk of wound breakdown, leak, or infection should not preclude radical surgical resection and aggressive adjuvant therapies in the setting of malignancy.

\section{Disclosures}

The authors have no disclosures or conflicts of interest.

\section{References}

1 Berger L, Luc R, Richard D. L'esthesioneuroepitheliome olfactif. Bull Assoc Fr Etud Cancer 1924;13:410-421

2 Liu JK, O’Neill B, Orlandi RR, Moscatello AL, Jensen RL, Couldwell WT. Endoscopic-assisted craniofacial resection of esthesioneuroblastoma: minimizing facial incisions-technical note and report of 3 cases. Minim Invasive Neurosurg 2003;46(05):310-315

3 Ferlito A, Rinaldo A, Rhys-Evans PH. Contemporary clinical commentary: esthesioneuroblastoma: an update on management of the neck. Laryngoscope 2003;113(11):1935-1938

4 Koka VN, Julieron M, Bourhis J, et al. Aesthesioneuroblastoma. J Laryngol Otol 1998;112(07):628-633

5 Loy AH, Reibel JF, Read PW, et al. Esthesioneuroblastoma: continued follow-up of a single institution's experience. Arch Otolaryngol Head Neck Surg 2006;132(02):134-138

6 Monroe AT, Hinerman RW, Amdur RJ, Morris CG, Mendenhall WM. Radiation therapy for esthesioneuroblastoma: rationale for elective neck irradiation. Head Neck 2003;25(07):529-534

7 Zafereo ME, Fakhri S, Prayson R, et al. Esthesioneuroblastoma: 25-year experience at a single institution. Otolaryngol Head Neck Surg 2008;138(04):452-458

8 Tajudeen BA, Arshi A, Suh JD, et al. Esthesioneuroblastoma: an update on the UCLA experience, 2002-2013. J Neurol Surg B Skull Base 2015;76(01):43-49

9 Herr MW, Sethi RKV, Chan A, et al. Esthesioneuroblastoma: an update on the Massachusetts eye and ear infirmary and Massachusetts general hospital experience with craniofacial resection. J Neurol Surg B Skull Base 2014;1(212):58-64

10 Folbe A, Herzallah I, Duvvuri U, et al. Endoscopic endonasal resection of esthesioneuroblastoma: a multicenter study. Am J Rhinol Allergy 2009;23(01):91-94

11 Polin RS, Sheehan JP, Chenelle AG, et al. The role of preoperative adjuvant treatment in the management of esthesioneuroblastoma: the University of Virginia experience. Neurosurgery 1998; 42(05):1029-1037

12 Komotar RJ, Starke RM, Raper DMS, Anand VK, Schwartz TH. Endoscopic endonasal compared with anterior craniofacial and combined cranionasal resection of esthesioneuroblastomas. World Neurosurg 2013;80(1-2):148-159

13 Hollen TR, Morris CG, Kirwan JM, et al. Esthesioneuroblastoma of the nasal cavity. Vestn Otorinolaringol 2015;38:84
14 Levine PA, McLean WC, Cantrell RW. Esthesioneuroblastoma: the University of Virginia experience 1960-1985. Laryngoscope 1986;96(07):742-746

15 McLean JN, Nunley SR, Klass C, Moore C, Müller S, Johnstone PA. Combined modality therapy of esthesioneuroblastoma. Otolaryngol Head Neck Surg 2007;136(06):998-1002

16 Devaiah AK, Andreoli MT. Treatment of esthesioneuroblastoma: a 16-year meta-analysis of 361 patients. Laryngoscope 2009; 119(07):1412-1416

17 Miyamoto RC, Gleich LL, Biddinger PW, Gluckman JL. Esthesioneuroblastoma and sinonasal undifferentiated carcinoma: impact of histological grading and clinical staging on survival and prognosis. Laryngoscope 2000;110(08):1262-1265

18 Gruber G, Laedrach K, Baumert B, Caversaccio M, Raveh J, Greiner R. Esthesioneuroblastoma: irradiation alone and surgery alone are not enough. Int J Radiat Oncol Biol Phys 2002;54(02):486-491

19 Foote RL, Morita A, Ebersold MJ, et al. Esthesioneuroblastoma: the role of adjuvant radiation therapy. Int J Radiat Oncol Biol Phys 1993;27(04):835-842

20 Dulguerov P, Allal AS, Calcaterra TC. Esthesioneuroblastoma: a meta-analysis and review. Lancet Oncol 2001;2(11):683-690

21 Song CM, Won TB, Lee CH, Kim DY, Rhee CS. Treatment modalities and outcomes of olfactory neuroblastoma. Laryngoscope 2012; 122(11):2389-2395

22 Levine PA, Gallagher R, Cantrell RW. Esthesioneuroblastoma: reflections of a 21-year experience. Laryngoscope 1999;109(10): 1539-1543

23 Resto VA, Eisele DW, Forastiere A, Zahurak M, Lee DJ, Westra WH. Esthesioneuroblastoma: the Johns Hopkins experience. Head Neck 2000;22(06):550-558

24 Eich HT, Müller RP, Micke O, Kocher M, Berthold F, Hero B. Esthesioneuroblastoma in childhood and adolescence. Better prognosis with multimodal treatment? Strahlenther Onkol 2005;181(06):378-384

25 Manthuruthil C, Lewis J, McLean C, Batra PS, Barnett SL. Endoscopic endonasal management of olfactory neuroblastoma: a retrospective analysis of 10 patients with quality of life measures. World Neurosurg 2016;90:1-5

26 Petruzzelli GJ, Howell JB, Pederson A, et al. Multidisciplinary treatment of olfactory neuroblastoma: patterns of failure and management of recurrence. Am J Otolaryngol 2015;36(04):547-553

27 Kadish S, Goodman M, Wang CC. Olfactory neuroblastoma. A clinical analysis of 17 cases. Cancer 1976;37(03):1571-1576

28 Patel SG, Singh B, Polluri A, et al. Craniofacial surgery for malignant skull base tumors: report of an international collaborative study. Cancer 2003;98(06):1179-1187

29 Dias FL, Sá GM, Kligerman J, et al. Complications of anterior craniofacial resection. Head Neck 1999;21(01):12-20

30 Ganly I, Patel SG, Singh B, et al. Complications of craniofacial resection for malignant tumors of the skull base: report of an International Collaborative Study. Head Neck 2005;27(06):445-451

31 Thorp BD, Sreenath SB, Ebert CS, Zanation AM. Endoscopic skull base reconstruction: a review and clinical case series of 152 vascularized flaps used for surgical skull base defects in the setting of intraoperative cerebrospinal fluid leak. Neurosurg Focus 2014;37(04):E4

32 Schmalbach CE, Webb DE, Weitzel EK. Anterior skull base reconstruction: a review of current techniques. Curr Opin Otolaryngol Head Neck Surg 2010;18(04):238-243

33 Chang DW, Langstein HN, Gupta A, et al. Reconstructive management of cranial base defects after tumor ablation. Plast Reconstr Surg 2001;107(06):1346-1355, discussion 1356-1357

34 Nameki H, KatoT, Nameki I, Ajimi Y. Selective reconstructive options for the anterior skull base. Int J Clin Oncol 2005;10(04):223-228 